\title{
Magnetic Resonance Electrical Impedance Tomography for Monitoring Electric Field Distribution During Tissue Electroporation
}

\author{
M. Kranjc, F. Bajd, I. Serša, and D. Miklavčič*
}

\begin{abstract}
Electroporation is a phenomenon caused by externally applied electric field of an adequate strength and duration to cells that results in the increase of cell membrane permeability to various molecules, which otherwise are deprived of transport mechanism. As accurate coverage of the tissue with a sufficiently large electric field presents one of the most important conditions for successful electroporation, applications based on electroporation would greatly benefit with a method of monitoring the electric field, especially if it could be done during the treatment. As the membrane electroporation is a consequence of an induced transmembrane potential which is directly proportional to the local electric field, we propose current density imaging (CDI) and magnetic resonance electrical impedance tomography (MREIT) techniques to measure the electric field distribution during electroporation. The experimental part of the study employs CDI with short high-voltage pulses, while the theoretical part of the study is based on numerical simulations of MREIT. A good agreement between experimental and numerical results was obtained, suggesting that CDI and MREIT can be used to determine the electric field during electric pulse delivery and that both of the methods can be of significant help in planning and monitoring of future electroporation based clinical applications.
\end{abstract}

Index Terms-Current density imaging (CDI), electroporation, magnetic resonance imaging (MRI), magnetic resonance impedance tomography (MREIT).

\section{INTRODUCTION}

A $\mathrm{N}$ exposure of a cell or tissue to an electric field of an adequate strength and duration leads to an increased cell membrane permeability. This phenomenon, termed electroporation, allows various otherwise nonpermeant molecules to cross the membrane and enter the cell [1]-[4].

When the strength of the applied electric field is below the irreversible electroporation threshold only transient structural changes can be attained and the cell membrane will reseal afterwards. This is termed reversible electroporation as cell preserves its viability [5]. On the contrary, irreversible electroporation leads to a cell death as the applied electric field is above

Manuscript received February 10, 2011; revised April 12, 2011; accepted April 19, 2011. Date of publication April 25, 2011; date of current version September 30, 2011. This work was supported by the Slovenian Research Agency (ARRS). Asterisk indicates corresponding author.

M. Kranjc is with the Faculty of Electrical Engineering, University of Ljubljana, SI-1000 Ljubljana, Slovenia (e-mail: matej.kranjc@fe.uni-lj.si).

F. Bajd and I. Serša are with the Institut "Jožef Štefan", Jamova cesta 39, SI-1000 Ljubljana, Slovenia and with the EN-FIST Centre of Excellence, SI-1000 Ljubljana, Slovenia (e-mail: franci.bajd@ijs.si; igor.sersa@ijs.si).

*D. Miklavčič is with the Faculty of Electrical Engineering, University of Ljubljana, SI-1000 Ljubljana, Slovenia (e-mail: damijan.miklavcic@fe.uni-lj. si).

Digital Object Identifier 10.1109/TMI.2011.2147328 the irreversible electroporation threshold and the cell does not regain its integrity [6]. An accurate coverage of the cell with a sufficiently large electric field therefore presents one of the most important conditions for successful electroporation [7], [8].

Applications such as electrochemotherapy (ECT) [9], [10], electroporation based gene transfer for gene therapy [11]-[13], DNA vaccination [14] and nonthermal irreversible electroporation ablation (NTIRE) [15], [16] are being successfully introduced into clinical practice. A new electroporation monitoring method that would allow direct measurement of the electric field strength within the sample would be of great importance for these applications. If possible the method should be performed in situ during the treatment. Another important aspect of the electroporation monitoring process is a possibility of input electroporation parameter adjustments according to the progress of the electroporation treatment as for now, optimal parameters for in vivo electroporation are determined using in vivo tests of permeabilization after the treatment [17] and by a mathematical modeling to determine the electric field distribution [18], [19]. Various methods of monitoring electroporation process were already suggested: electrical conductivity measurement by electrical impedance tomography (EIT) [20], [21], current and voltage measurements of delivered pulses [22] and recently magnetic resonance imaging (MRI) [23], [24]. Although these approaches, if further developed, might be interesting, they are either unable to monitor the process during pulse delivery in case of EIT and MRI approach or they can deliver false results due to lack of measurements in case of current and voltage measurements of delivered pulses.

Tissue is an electrically conducting material and it can be exposed to an electric field by injecting electric current by an electric pulse generator via electrodes. When an electric current is injected into an electrically conductive body such as a tissue, the magnetic field density $\mathbf{B}=\left(B_{x}, B_{y}, B_{z}\right)$ and the electric current density $\mathbf{J}=\left(J_{x}, J_{y}, J_{z}\right)$ are established inside this body. The electric current density inside conductive watery samples can be obtained using current density imaging technique (CDI) by measuring magnetic field density $\mathbf{B}$ with MRI and solving Ampere's law equation $\mathbf{J}=(1) /\left(\mu_{0}\right) \nabla \times \mathbf{B}$ [25]-[27]. One of many CDI applications is also magnetic resonance electrical impedance tomography (MREIT), a technique used for reconstruction of electrical conductivity inside a conducting body by means of current density (J-based MREIT) [28]-[31] or one component of magnetic flux density- $B_{z}\left(B_{z}\right.$-based MREIT) [32], [33]. The main problem of $\mathbf{J}$-based MREIT is in a difficulty to image the sample in different perpendicular orientations in order to obtain all three components of $\mathbf{B}$ and $\mathbf{J}$. MRI namely allows measurement of only one $\mathbf{B}$ component at a time, 
i.e., the component in the direction of the main magnetic field. The sample reorientation may result in a pixel misalignments and a sample deformation. For this reason most in vivo studies [34]-[36] have been usually done with $B_{z}$-based MREIT in which conductivity distribution is obtained without the sample rotation. When both, the electrical conductivity and the current density are obtained, the electric field distribution inside the sample can be calculated using Ohm's law. As the membrane electroporation is a consequence of an induced transmembrane potential which is directly proportional to the local electric field [8], [37], CDI and MREIT techniques could be useful techniques to monitor the electroporation process by measuring the electric field distribution during electroporation.

CDI and MREIT were designed for diagnostic purposes in which the injected current is not producing any significant effect on the tissue. For that reason the injected current is limited to a few milliampere or even less, which corresponds to voltage pulses of only few volts. There are few reports published where in CDI voltage pluses of higher amplitudes were used; for example in CDI of mice tumors $160 \mathrm{~V}$ electric pulses were used [38], however, there is no report on CDI where electric pulses of voltages that are normally used in electroporation would be used. In electrochemotherapy and non-thermal irreversible electroporation ablation the electric pulses can be up to $3000 \mathrm{~V}$ depending on electrodes geometry and a distance between the electrodes.

The aim of this work was to study feasibility of MREIT to monitor electric field distribution during tissue electroporation. The experimental part of the study employs CDI with short high-voltage pulses, as are normally used in electroporation based clinical applications such as electrochemotherapy and nonthermal irreversible electroporation ablation, while the theoretical part of the study is based on numerical simulations of MREIT. All experiments were performed on two different phantoms, a homogeneous and a heterogeneous phantom. The homogeneous phantom was made of agar with a single electrical conductivity, which resembled the conductivity of a typical tumor, while the heterogeneous phantom was made of two agars, with two different electrical conductivities; one resembling the conductivity of a tumor and the other resembling the conductivity of a liver. They were exposed to different electroporation pulse sequences with different number of pulses of different amplitudes. The current density distribution inside the phantoms was measured by CDI and the electric field distribution inside the phantom was calculated by the MREIT algorithm. For comparison with the experimental results a numerical model of the phantom was constructed using the finite element method.

\section{MATERIALS AND MethodS}

\section{A. Experimental Setup}

Cylindrically shaped homogeneous and heterogeneous phantoms measuring $21 \mathrm{~mm}$ in radius and $2 \mathrm{~mm}$ in height made of an agar mixture were placed in the acrylic glass container as shown in Fig. 1. The agar mixture was made of agar powder (Kemika, Zagreb, Croatia), $0.9 \% \mathrm{NaCl}$ saline solution (B. Braun, Melsungen, Germany), and distilled deionized water (B. Braun, Melsungen, Germany). The homogeneous phantom

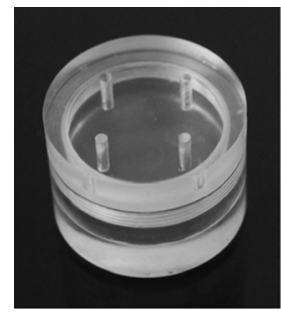

(a)

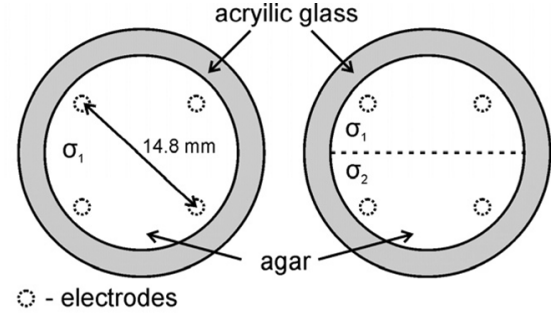

(b) (c)
Fig. 1. Phantom used in the study was made of the acrylic glass container with four holes for electrodes (a). The phantom was used in two different arrangements: in the homogeneous arrangement (b) it was filled with one agar type having homogeneous electrical conductivity, while in the heterogeneous arrangement (c) it was filled with two agar types with two different electrical conductivities, each filing half of the cylinder.

was made of a single cylindrically shaped agar mixture with electrical conductivity $\left(\sigma_{1}=0.23 \mathrm{~S} / \mathrm{m}\right)$ as shown in Fig. 1(b), while the heterogeneous phantom was made of two half cylindrically shaped agar mixtures - each of them with its own electrical conductivity ( $\sigma_{1}=0.23 \mathrm{~S} / \mathrm{m}$ and $\sigma_{2}=0.05 \mathrm{~S} / \mathrm{m}$ ) as shown in Fig. 1(c). The first conductivity resembles a tumor conductivity and the second conductivity resembles a liver conductivity [39]-[41]. Electrical conductivity of agar mixtures was measured by Conductivity meter MA 5950 (Metrel, Horjul, Slovenia). Four cylindrically shaped electrodes-needles measuring $1 \mathrm{~mm}$ in radius made of platinum-iridium alloy were inserted through the cover of the acrylic glass container into the phantom as shown in Fig. 1(a). Distance between the diagonal electrodes from the center to the center was $14.8 \mathrm{~mm}$.

Electric pulse generator used for delivering electroporation pulses into the phantom was customized Cliniporator Vitae (IGEA, Carpi, Italy). The Cliniporator Vitae device is a pulse generator with six independently controlled and electrically insulated outputs each providing rectangular pulses with amplitudes up to $3000 \mathrm{~V}$ and $50 \mathrm{~A}$ maximum current. The generator is also capable of measuring the output voltage at $3 \%$ precision. Four of the outputs were connected to four electrodes inserted in the phantom. The outputs were also measured with an oscilloscope (WavePro 7300A, LeCroy, Chestnut Ridge, NY) and current probe (AP015, LeCroy, Chestnut Ridge, NY) to obtain the current amplitude of the delivered pulses $\left(I_{\mathrm{OSC}}\right)$. The trigger input of the generator was connected to the MRI control unit and synchronized with the CDI pulse sequence.

Homogenous and heterogeneous phantoms were exposed to sequences of electric pulses, which are commonly used in electroporation applications [42]. The electric pulses were delivered between the diagonal electrodes of the sample in sequences of either one, two, four, or eight $100-\mu \mathrm{s}$-long pulses separated by $100 \mu \mathrm{s}$ intervals. Every sequence of pulses was delivered three times, each time with a different pulse amplitude $\left(U_{\text {app }}\right)$. In experiments on homogeneous phantoms, voltage amplitudes of the applied pulses were $U_{\mathrm{app}}=1000 \mathrm{~V}, 1500 \mathrm{~V}$, and $2000 \mathrm{~V}$, while in experiments on heterogeneous phantoms the amplitudes were somewhat higher $U_{\mathrm{app}}=1000 \mathrm{~V}, 2000 \mathrm{~V}$, and $3000 \mathrm{~V}$ due to its lower electrical conductivity compared to the homogeneous phantom. All experiments were repeated three times. 


\section{B. Current Density Imaging}

Electric current distribution in all the phantoms was imaged by the two-shot RARE current density magnetic resonance imaging sequence [43]. The sequence consists of two parts, the current encoding part and the imaging part based on the RARE MRI sequence [44]. During the current encoding part, which is essentially the conventional spin-echo sequence with superimposed electric pulses, the electroporation train of high-voltage electric pulses was executed in the interval between the excitation $90^{\circ} \mathrm{RF}$ pulse and the refocusing $180^{\circ} \mathrm{RF}$ pulse. The electric pulses induced a phase shift in the nuclear magnetic resonance (NMR) signal that is proportional to the time integral of the applied electric pulses. The phase shift must be preserved during the imaging part of the sequence and stored in the phase of the MR image. This is in the two-shot RARE CDI sequence achieved by signal co-addition of two RARE sequences having phases of the refocusing RF pulses $90^{\circ}$ apart. Current density in the phantom was calculated using the Ampere law

$$
\mathbf{J}=\frac{1}{\mu_{0}} \nabla \times \mathbf{B}
$$

from current induced phase shifts $\varphi$ stored in the acquired images

$$
\varphi=\gamma t_{c} B_{z}
$$

Here $\gamma$ is the proton gyromagnetic ratio and $t_{\mathrm{c}}$ is the total duration of the applied electric pulses. In the presented experiments the phantom geometry was such that currents were flowing predominately in the direction perpendicular to the electrodes. Therefore, the magnetic field in the central axial slice through the phantom had nonzero component in the direction perpendicular to the imaging slice, i.e., $B_{\mathrm{z}}$, and negligible in-slice components $\left(B_{\mathrm{x}}, B_{\mathrm{y}}\right)$. Under these conditions current density calculation simplifies to

$$
\mathbf{J}=\frac{1}{\mu_{0}}\left(\frac{\partial B_{z}}{\partial y},-\frac{\partial B_{z}}{\partial x}, 0\right)
$$

It should be noted here that such slice/sample/electrode arrangement does not require sample reorientation to perpendicular orientations that is usually inevitable for other arrangements in order to acquire other components of current induced magnetic field changes.

MR imaging was performed on a TecMag NMR spectrometer connected to an Oxford 2.35T horizontal bore superconducting magnet. The MRI system was equipped with Bruker microimaging accessories with maximum gradients of $250 \mathrm{mT} / \mathrm{m}$. The phantoms were inserted in the $25 \mathrm{~mm}$ RF probe and connected to the electric pulse generator using cables including low-pass filters to avoid possible RF disturbances in the NMR signal. MR images of current induced magnetic field changes were acquired using the two-shot RARE CDI sequence as shown in Fig. 2 with parameters: field of view (FOV) $30 \mathrm{~mm}$, imaging matrix $64 \times 64$, inter-echo delay $2.64 \mathrm{~ms}$, echo time of the current encoding period $20 \mathrm{~ms}$ and the time interval between the two RARE signal acquisitions was $10 \mathrm{~s}$. As the phantom

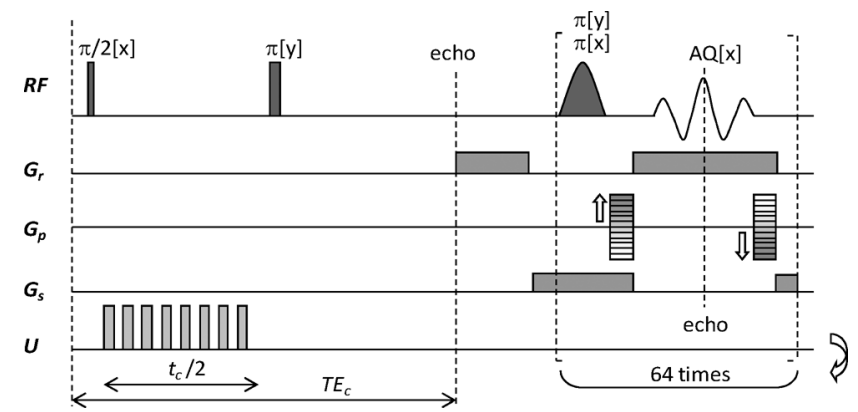

Fig. 2. Two-shot RARE CDI sequence that was used to acquire images of current induced magnetic field changes.

was flat, i.e., in a form of an excited slice, slice excitation RF pulses were not needed and they were replaced by nonselective RF pulses that excited the entire phantom, while the imaging plane coincided with the "phantom slice."

\section{Electric Field Reconstruction}

Electric field was reconstructed by means of $\mathbf{J}$ obtained with CDI method, application of the $\mathbf{J}$-substitution algorithm for reconstruction of conductivity distribution [28], [32], [45], [46] and Ohm's law for calculation of electric field distribution. A mathematical model of the phantom body $\Omega_{\mathrm{p}}$ surrounded with the outer boundary $\partial \Omega_{\mathrm{p}_{-} \mathrm{o}}$ was build. Four electrodes were modeled as four inner boundaries $\partial \Omega_{\mathrm{p} \_\mathrm{e}}$ inside the body $\Omega_{\mathrm{p}}$.

The Corresponding voltage $u$ satisfies the boundary value problem in any given conductivity $\sigma$ of the model

$$
\nabla \cdot(\sigma \nabla u)=0 \quad \text { in } \Omega_{\mathrm{p}}
$$

with the corresponding Neumann boundary condition on the phantom outer boundary $\partial \Omega_{\text {p_o }}$

$$
\sigma \frac{\partial u}{\partial n_{\mathrm{p}}}=0 \quad \text { on } \partial \Omega_{\mathrm{p} \_\mathrm{o}}
$$

where $n_{\mathrm{p}}$ denotes the exterior normal vector to the boundary $\partial \Omega_{\mathrm{p} \_o}$ and Dirichlet boundary condition on the inner-electrodes boundary $\partial \Omega_{\mathrm{p} \_\mathrm{e}}$

$$
u=V \quad \text { on } \partial \Omega_{\mathrm{p} \_\mathrm{e}}
$$

where $V$ is the voltage on the electrodes measured by the generator.

Both voltage $u$ and electrical conductivity $\sigma$ in (4) are unknown variables. Equations (4), (5), and (6) are solved by the iterative scheme yielding the correct value of $\sigma$

$$
\begin{aligned}
\nabla \cdot\left(\sigma^{k} \nabla u^{k}\right) & =0 & & \text { in } \Omega_{\mathrm{p}} \\
\sigma^{k} \frac{\partial u^{k}}{\partial n_{\mathrm{p}}} & =0 & & \text { on } \partial \Omega_{\mathrm{p} \_\mathrm{o}} \\
u^{k} & =V & & \text { on } \partial \Omega_{\mathrm{p} \_\mathrm{e}}
\end{aligned}
$$

All iterative schemes in this study started with $\sigma^{0}=1(k=0)$. After solving (7), (8), and (9) the solution $u^{k}$ was used in

$$
\sigma^{k+1}=\frac{\left|\mathbf{J}_{\mathrm{CDI}}\right|}{\left|\nabla u^{k}\right|}
$$


where $\sigma^{k+1}$ is the new conductivity and $\mathbf{J}_{\mathrm{CDI}}$ is the current density obtained by the CDI method. The iterative scheme lasts until the relative difference between two successive $\sigma$ falls below $\varepsilon$ (in our study $\varepsilon$ had value of 0.01 )

$$
\frac{\left\|\sigma^{k}-\sigma^{k-1}\right\|}{\left\|\sigma^{k}\right\|}<\varepsilon
$$

Electric field distribution $\mathbf{E}_{\text {MREIT }}$ can then be calculated using Ohm's law

$$
\mathbf{E}_{\text {MREIT }}=\frac{\mathbf{J}_{\mathrm{CDI}}}{\sigma^{k}} .
$$

Relative error $e$ of the obtained electric field distribution was calculated using

$$
e=\frac{\left|\mathbf{E}_{\text {num }}-\mathbf{E}_{\text {MREIT }}\right|}{\left|\mathbf{E}_{\text {num }}\right|}
$$

where $\boldsymbol{E}_{\text {num }}$ is the electric field distribution obtained by solving the numerical model.

It should be noted that different conductivity distributions may correspond to the same current density distribution in a heterogeneous sample due to nonuniqueness [47]. For this reason, the heterogeneous phantom was measured with two different sequences of electric pulses that satisfy the condition

$$
\left|\mathbf{J}_{\mathrm{CDI} \_1} \times \mathbf{J}_{\mathrm{CDI} \_2}\right| \neq 0
$$

here $\mathbf{J}_{\mathrm{CDI} \_1}$ and $\mathbf{J}_{\mathrm{CDI} \_2}$ are the two current density distributions that result from the two different pulse sequences. Therefore pulse sequences were delivered between both diagonal pairs of electrodes.

The finite element method was used to solve the problem in (7), (8), and (9). All calculations of the mathematical model were performed with the numerical computational environment Matlab 2010a (Mathworks, Natick, MA) and its Partial Differential Equation Toolbox on a desktop PC (Windows 7, 2.66 $\mathrm{GHz}, 4 \mathrm{~GB}$ RAM). The calculations took on average only a few seconds.

\section{Numerical Model}

Experimental results obtained by CDI and MREIT were compared also to two numerical models: one for the homogeneous and the other for the heterogeneous phantom. The electric field distribution $\left(\mathbf{E}_{\text {num }}\right)$, corresponding current density distribution $\left(\mathbf{J}_{\text {num }}\right)$ and its spatial integral, i.e., the net electric current $\left(I_{\text {num }}\right)$ in numerical models were obtained by solving (4), (5), and (6) using known conductivities of the homogeneous $\left(\sigma_{1}\right)$ and heterogeneous phantoms $\left(\sigma_{1}\right.$ and $\left.\sigma_{2}\right)$. The numerical model was solved using a finite element method with the commercial finite element software package COMSOL Multiphysics 3.5a (COMSOL AB, Stockholm, Sweden) running on the same desktop PC as noted previously. The mesh of the numerical model consisted of 6752 triangular elements.

\section{RESULTS}

When the phantom was exposed to electroporation pulses an electric current density and electric field were established inside the phantom. The former was successfully measured by

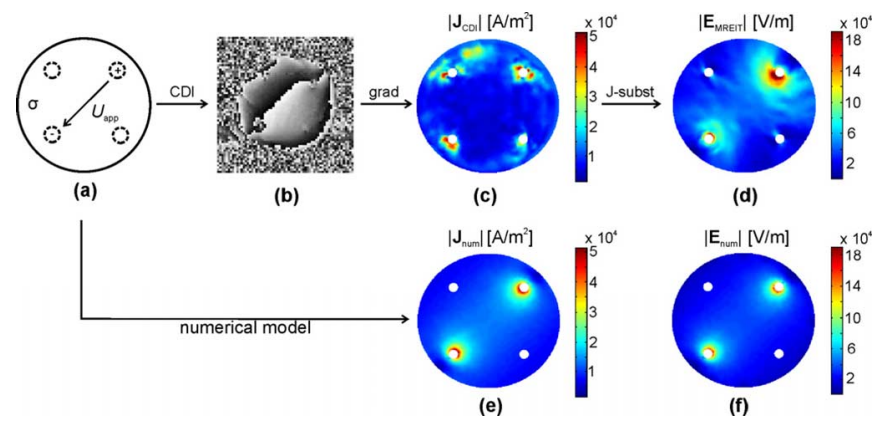

Fig. 3. Homogeneous phantom exposed to four high voltage pulses of $1000 \mathrm{~V}$ delivered between diagonal electrodes (a). The initial phase image (b) was acquired by the two-shot RARE based CDI sequence. The current density distribution (c) in the phantom was calculated from the phase image using (3). Finally, the electric field distribution (d) was calculated using J-substitution algorithm from the current density distribution. Numerical model results of current density distribution (e) and electric field distribution (f) in the phantom are shown below the corresponding experimental results.

CDI method, while the later was calculated from the CDI data using the MREIT J-substitution algorithm. Both results are presented in Fig. 3, which corresponds to the experiment on the homogeneous phantom exposed to four electroporation pulses of $1000 \mathrm{~V}$. Current densities and electric field distributions in the homogeneous phantom were also successfully and reproducibly obtained for other pulse parameters (data not shown). Fig. 3 includes also results of the numerical model for the same phantom. The numerical results are in a good agreement with the experimental results as we obtained $7.8 \%$ relative error for homogeneous phantoms when we compared experimental with numerical results.

Current densities and electric field distribution obtained by CDI and MREIT in the heterogeneous phantom exposed to four electric pulses of $1000 \mathrm{~V}$ are shown in Fig. 4 along with the numerical model results. Current densities and electric field distributions in the heterogeneous phantoms were also successfully and reproducibly obtained for other pulse parameters (data not shown). When we compared experimental with numerical results, we obtained $9.2 \%$ and $22 \%$ relative error for more conductive half $\left(\sigma_{1}\right)$ and less conductive half $\left(\sigma_{2}\right)$ of the phantoms, respectively.

Measured electric currents during applied electric pulses by a current probe and by CDI are presented in Table I for the homogeneous and heterogeneous phantom together with the numerical model results.

In order to evaluate electric field distribution obtained with J-substitution, electric field diagonally across the phantom was compared with the electric field obtained by solving numerical model as shown in Fig. 5 for heterogeneous phantom.

Phantoms were exposed to different sequences of electric pulses which differed in number of applied electric pulses and by their amplitudes. Electric field distributions for the homogeneous phantom exposed to different number of pulses $(N=$ $1,2,4,8)$ with the amplitude of $1000 \mathrm{~V}$ are shown in Fig. 6. When we compared experimental with numerical results, we obtained an average relative error of $9.7 \%, 7.9 \%, 7.3 \%$, and $6.2 \%$ for $\mathrm{N}=1,2,4$, and 8 , respectively.

Electric field distributions in the homogeneous phantom for different amplitudes pulses $\left(U_{\mathrm{app}}=1000,1500,2000 \mathrm{~V}\right)$ of 


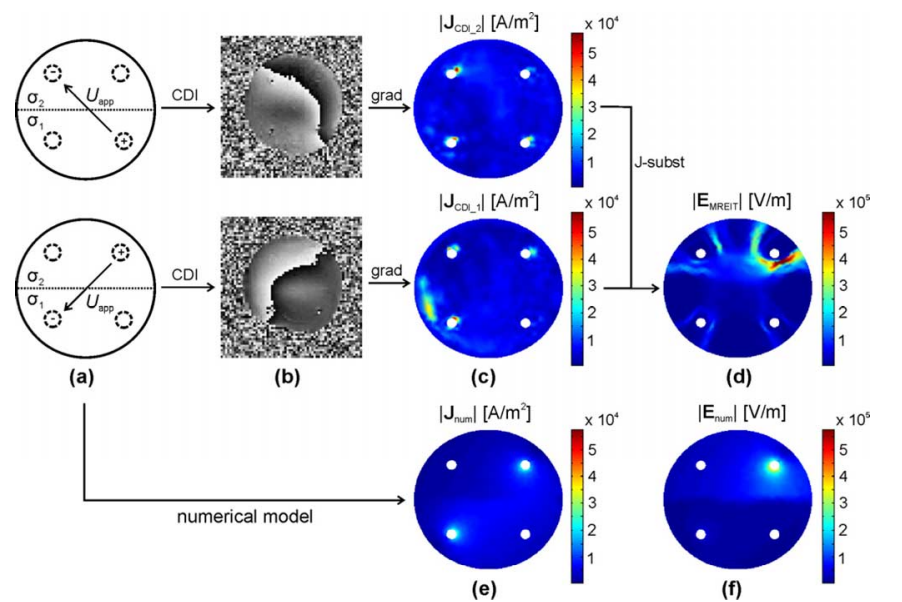

Fig. 4. Heterogeneous phantom exposed to four electric pulses of $1000 \mathrm{~V}$ delivered between diagonal electrodes (a). Initial phase images (b) were acquired by the two-shot RARE CDI sequence. Current density distributions (c) in the phantom were obtained by applying (3) to phase images. Finally, the electric field distribution (d) was calculated using the J-substitution algorithm from both current density distributions. Due to heterogeneous electrical conductivity of the phantom two different current density distributions were needed to obtain a unique conductivity distribution. Corresponding numerical model results of current density distribution (e) and of electric field (f) distribution in the phantom are shown below experimental.

TABLE I

COMPARISON OF TWO EXPERIMENTAL $\left(I_{\text {osc }}\right.$ AND $\left.I_{\text {int }}\right)$ AND ONE Numerical $\left(I_{\text {num }}\right)$ APPROACH IN ASSESSMENT OF ElECTRIC CURRENTS IN Electroporation. $I_{\mathrm{osc}}$ Presents Electric CURRENT MEAsured With the Oscilloscope During Pulse Delivery, $I_{\text {int }}$ Is a Solution of SuRface InTEGRAL OVER MEASURED CURRENT DENSITY ObTAINED By CDI AND $I_{\text {num }}$ Is A SOlution of the Numerical Model. Results of THREe EXPERIMENTS ARE PRESENTED AS MEANS \pm STANDARD DEVIATION

\begin{tabular}{ccccccc}
\hline \hline & \multicolumn{3}{c}{ Homogeneous phantom } & \multicolumn{3}{c}{ Heterogeneous phantom } \\
& \multicolumn{3}{c}{$U_{\text {app }}[\mathrm{V}]$} & \multicolumn{3}{c}{$U_{\text {app }}[\mathrm{V}]$} \\
& 1000 & 1500 & 2000 & 1000 & 2000 & 3000 \\
\hline & $3.3 \pm$ & $5.0 \pm$ & $8.9 \pm$ & $1.1 \pm$ & $2.1 \pm$ & $4.0 \pm$ \\
$I_{\text {osc }}[\mathrm{A}]$ & 0.1 & 0.2 & 1.1 & 0.1 & 0.1 & 0.6 \\
& & & & & & \\
& & & & & \\
$I_{\text {int }}[\mathrm{A}]$ & $2.9 \pm$ & $4.6 \pm$ & $7.2 \pm$ & $1.5 \pm$ & $2.6 \pm$ & $3.8 \pm$ \\
& 0.3 & 0.3 & 1.8 & 0.1 & 0.2 & 0.5 \\
$I_{\text {num }}[\mathrm{A}]$ & 3.2 & 4.9 & 6.5 & 1.2 & 2.3 & 3.5 \\
\hline \hline
\end{tabular}

four electric are shown in Fig. 7. When we compared experimental with numerical results, we obtained an average relative error of $6.4 \%, 7.1 \%$, and $8.0 \%$ for $U_{\text {app }}=1000 \mathrm{~V}, 1500 \mathrm{~V}$, and $2000 \mathrm{~V}$, respectively.

\section{DISCUSSION}

The aim of our study was to investigate CDI and MREIT techniques for its feasibility to monitor the process of electroporation by measuring electric field distribution during electroporation. Experiments on homogeneous and heterogeneous phantoms were performed and compared to the results of the numerical model. Different number of pulses and of their amplitudes were used in separate experiments to evaluate feasibility of the methods to determine electric field distribution during the electroporation. Electric current flowing between

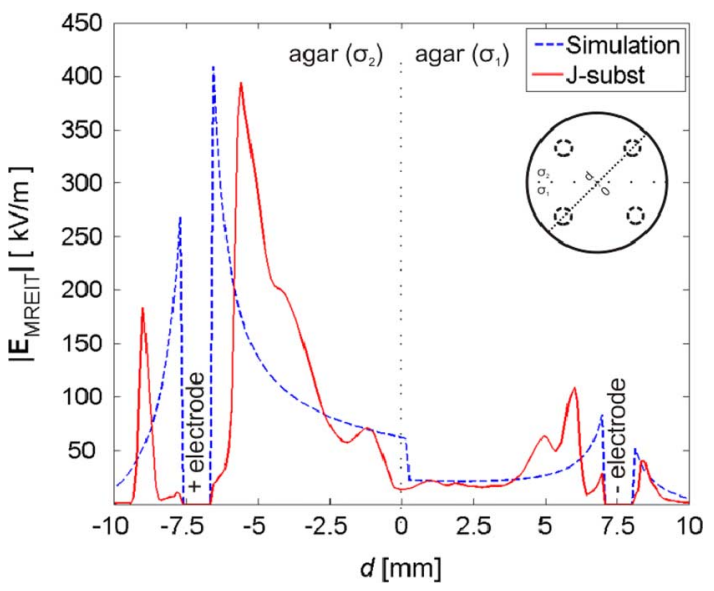

Fig. 5. Electric field diagonally across the heterogeneous phantom (see dashed line in the right insert of the figure) calculated with the J-substitution algorithm (solid red line) and electric field calculated by the numerical model (blue dashed line). The phantom was exposed to four pulses of $1000 \mathrm{~V}$. On the $x$ axis $d$ presents distance from the center to the border of the phantom.

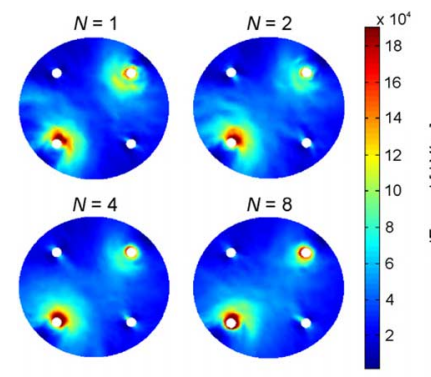

(a)

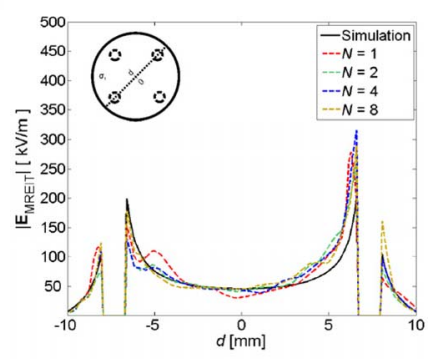

(b)
Fig. 6. Electric field distribution (a) and electric field diagonally across the homogeneous phantom (b) for different number of electric pulses. The phantoms were exposed to one, two, four, or eight pulses of $1000 \mathrm{~V}$.

the electrodes during pulse sequences was obtained with three different approaches, two experimental and one numerical. Results presented in Table I confirm good agreement between the approaches in both phantoms used and for most of the applied voltages. A minor discrepancy was observed between the homogeneous phantom exposed to pulses of $2000 \mathrm{~V}$ and the heterogeneous phantom exposed to pulses of $3000 \mathrm{~V}$ due to exceedingly high current in agar of the phantoms, resulting in its deterioration due to excessive heating and consequently in higher electrical conductivity. As shown in Fig. 5, experimentally and numerically obtained electric field profiles diagonally across the phantoms are in a good agreement. The largest disagreement between experimental and numerical results was observed in areas close to electrodes. Electrodes manly cause large distortions in the magnetic field and significant susceptibility artifacts. Magnetic field distortion around electrodes does not represent considerable problem to monitor electric field distribution during electroporation as the area between the electrodes is more important for efficient electroporation. CDI and MREIT allowed determination of the electric field distribution for all pulse sequences used in our study. The experiments showed that the electric field intensity and its distribution are independent of the number of electric pulses (Fig. 6) while solely its distribution is independent of their amplitude (Fig. 7). 


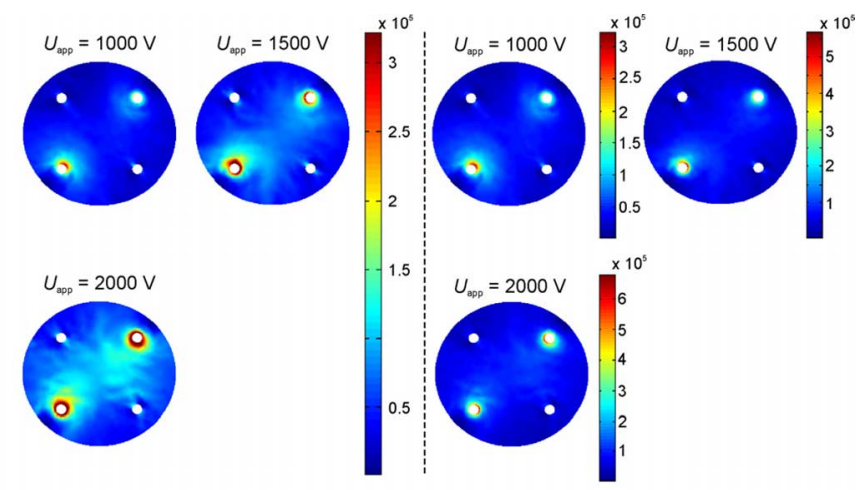

(a)

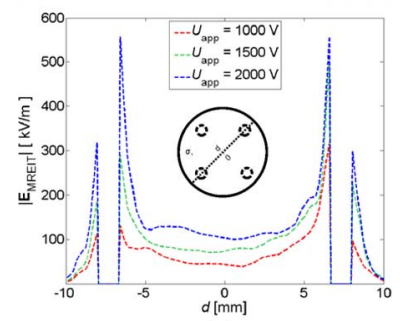

(c) (b)

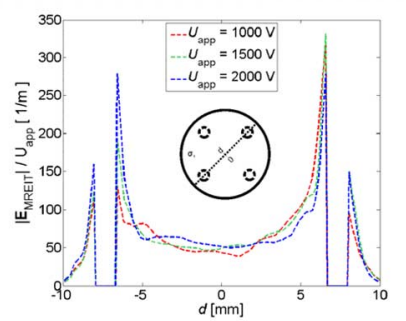

(d)

Fig. 7. Electric field distributions (a), (b) and electric fields diagonally across the phantoms (c) with homogenous electrical conductivity exposed to sequences of four electric pulses of different amplitudes $\left(U_{\text {app }}=1000 \mathrm{~V}, 1500 \mathrm{~V}, 2000\right.$ V). Electric field distributions on the upper left (a) are scaled to the same range while three distributions on the upper right (b) are scaled according to their maximum and minimum value of each distribution. Electric fields diagonally across the phantoms normalized to the amplitude of applied pulses $\left(\left|\mathbf{E}_{\mathrm{MREIT}}\right| / U_{\mathrm{app}}\right)$ are also presented (d).

As already mentioned in the introduction, different attempts to monitor electroporation process were already proposed. Similar to MREIT, EIT is used for reconstruction of conductivity images only that voltage and current measurements of the EIT are limited to the boundaries of the object. Difficulties associated with the use of EIT to monitor electroporation process [20] are identical to those of using EIT to image tissue in general; numerous additional electrodes are required, sensitivity, spatial resolution and accuracy are low, while noise is high. Recent published report on MRI of nonthermal irreversible electroporation in vegetable tissue is limited in observation of only irreversible electroporation where the damage to the cellular membrane and the consequent release of intracellular content can be detected by comparing MRI images of different modalities $\left(T_{1^{-}}\right.$, $T_{2}$-weighted, FLAIR or STIR) acquired before and after the application of NTIRE pulses [23]. Similar method of comparing MRI images of different modalities was used to observe irreversible electroporation of liver tissues by detecting local fluid accumulation owing to transient permeabilization of blood vessels, as suggested by authors [24]. Main disadvantage of all of the above mentioned methods is the incapability to monitor electroporation during pulse delivery. This does not apply to the method of current and voltage measurement of delivered pulses described in [22], although this method inherits the main restraint of conductivity evaluation methods that are based on voltage/current measurements. Monitoring of voltage and current between only one pair of electrodes at a time can led to false conductivity and to an inaccurate electric field distribution

calculation due to lack of information on tissue heterogeneity between the electrodes. More accurate monitoring of electroporation during pulse delivery can be accomplished with acquiring current density information during pulse delivery and its transformation to the electric field distribution by means of proposed CDI and MREIT methods. As single two-shot RARE CDI sequence used in this study takes about $20 \mathrm{~s}$ and MREIT J-substitution algorithm additional few seconds, the electric field distribution can then be obtained in less than a minute after the beginning of pulse delivery in case of heterogeneous tissues. This time could be additionally reduced in future by means of faster CDI sequences.

The main limitation of our study lies in the phantom substance - agar, which became deteriorated after long exposure to high-voltage pulses resulting in measurement failure. A technical limitation of the MREIT algorithm used in this study is also inability of anisotropic conductivity measurement. For that reason implementation of more advanced MREIT methods [48] will be examined in our future studies. The difficulty of using MREIT to monitor electric field distribution during electroporation in a clinical environment is associated with the limited capability of MRI scanners for their use in interventional procedures, although recently a report on MREIT with an open magnet systems was published [49]. A question that needs answering in the near future is whether it is possible to obtain an accurate current density distribution in CDI from only one $\mathbf{B}$ component. However, it was already reported that longitudinal current density component $J_{7}$ in a transversal imaging slice ( $x y$-plane) can be successfully measured when current flows mainly in the transversal direction, i.e., the case when longitudinal electrodes, similar those used in ECT and NTIRE experiments, are used [50], [51].

NTIRE and ECT treatments are two electroporation applications that would probably benefit most with the implementation of CDI and MREIT. NTIRE is a new minimally invasive surgical technique for tissue ablation in which electroporation pulses form nanoscale defects in the cell membrane that lead to cell death, while ECT, a relatively new approach to cancer treatment and the most established in vivo application of electroporation, treats tumors by increasing the uptake of chemotherapeutic drugs into the tumor cells by exposing it to the electric field. Adequate local electric field distribution is crucial for success of both therapies. The treatment planning for ECT already proved to have a great potential in clinical use of ECT in treatment of tumors [52]. However, its applicability is currently limited due to uncertain conductivity values of treated areas, especially within the tumor where heterogeneous conductivity was already observed [53], resulting in obtaining inappropriate electrodes position and electric pulse parameters. Another concern is electrodes positioning during the ECT treatment procedure. Namely, it is difficult to insert electrodes precisely according to the treatment plan [54]. An imprecise placement of the electrodes can thus result in an inadequate electric field coverage of the treated area and therefore a treatment failure. Monitoring of the electric field distribution during ECT and NTIRE would enable detection of an insufficient electric field coverage before the end of the treatment, thus increasing and assuring the effectiveness of both methods. 


\section{CONCLUSION}

Monitoring electric field distribution during tissue electroporation by means of CDI and MREIT is described and investigated both experimentally and numerically on homogeneous and heterogeneous phantom with electric properties similar to a liver and a tumor. Synchronization of electroporation pulses with the CDI sequence enabled imaging of current densities during electroporation. This was followed by calculation of the electric field distribution using the MREIT J-substitution method. A good agreement between experimental and numerical results was obtained, suggesting that CDI and MREIT can be used to determine the electric field during electric pulse delivery and that both of the methods can be of significant help in planning and monitoring of future electroporation based clinical applications such as electrochemotherapy, nonthermal irreversible electroporation ablation and electroporation based gene transfer for gene therapy.

\section{ACKNOWLEDGMENT}

The authors would like to thank Prof. Dr. G. Serša for valuable comments and suggestions during the preparation of the manuscript.

\section{REFERENCES}

[1] L. M. Mir and S. Orlowski, "Mechanisms of electrochemotherapy," Adv. Drug Delivery Rev., vol. 35, no. 1, pp. 107-118, 1999.

[2] E. Neumann, M. Schaeferridder, Y. Wang, and P. Hofschneider, "Gene-transfer into mouse lyoma cells by electroporation in high electric-fields," EMBO J., vol. 1, no. 7, pp. 841-845, 1982.

[3] S. Orlowski, J. Belehradek, C. Paoletti, and L. M. Mir, "Transient electropermeabilization of cells in culture. Increase of the cytotoxicity of anticancer drugs," Biochem. Pharmacol., vol. 37, no. 24, pp. $4727-4733,1988$.

[4] G. Serša, M. Čemažar, and D. Miklavčič, "Antitumor effectiveness of electrochemotherapy with cis-diamminedichloroplatinum(II) in mice," Cancer Res., vol. 55, no. 15, pp. 3450-3455, 1995.

[5] A. M. Lebar, G. Sersa, S. Kranjc, A. Groselj, and D. Miklavcic, "Optimisation of pulse parameters in vitro for in vivo electrochemotherapy," Anticancer Res., vol. 22, no. 3, pp. 1731-1736, 2002.

[6] R. V. Davalos, I. L. M. Mir, and B. Rubinsky, "Tissue ablation with irreversible electroporation," Ann. Biomed. Eng., vol. 33, no. 2, pp. 223-231, 2005.

[7] D. Miklavcic, S. Corovic, G. Pucihar, and N. Pavselj, "Importance of tumour coverage by sufficiently high local electric field for effective electrochemotherapy," Eur. J. Cancer Suppl., vol. 4, no. 11, pp. 45-51, 2006.

[8] D. Miklavcic, K. Beravs, D. Semrov, M. Cemazar, F. Demsar, and G. Sersa, "The importance of electric field distribution for effective in vivo electroporation of tissues," Biophys. J., vol. 74, no. 5, pp. 2152-2158, 1998.

[9] M. Marty et al., "Electrochemotherapy-An easy, highly effective and safe treatment of cutaneous and subcutaneous metastases: Results of ESOPE study," Eur. J. Cancer Suppl., vol. 4, no. 11, pp. 3-13, 2006.

[10] G. Sersa and D. Miklavcic, "Electrochemotherapy of tumours," J. Vis. Experiments, no. 22, 2008.

[11] F. Andre et al., "Efficiency of high- and low-voltage pulse combinations for gene electrotransfer in muscle, liver, tumor, and skin," Human Gene Therapy, vol. 19, no. 11, pp. 1261-1271, 2008.

[12] L. Heller and R. Heller, "Electroporation gene therapy preclinical and clinical trials for melanoma," Current Gene Therapy, vol. 10, no. 4, pp. 312-317, 2010

[13] A. I. Daud et al., "Phase I trial of interleukin-12 plasmid electroporation in patients with metastatic melanoma," J. Clin. Oncol., vol. 26, no. 36, pp. 5896-5903, 2008.

[14] L. Zhang, G. Widera, and D. Rabussay, "Enhancement of the effectiveness of electroporation-augmented cutaneous DNA vaccination by a particulate adjuvant," in Bioelectrochemistry, Amsterdam, The Netherlands, 2004, vol. 63, no. 1-2, pp. 369-373.
[15] P. A. Garcia et al., "Non-thermal irreversible electroporation (N-TIRE) and adjuvant fractionated radiotherapeutic multimodal therapy for intracranial malignant glioma in a canine patient," Technol. Cancer Res. Treatment, vol. 10, no. 1, pp. 73-83, 2011.

[16] J. Rubinsky, G. Onik, P. Mikus, and B. Rubinsky, "Optimal parameters for the destruction of prostate cancer using irreversible electroporation," J. Urol., vol. 180, no. 6, pp. 2668-2674, 2008.

[17] J. Gehl and L. M. Mir, "Determination of optimal parameters for in vivo gene transfer by electroporation, using a rapid in vivo test for cell permeabilization," Biochem. Biophys. Res. Commun., vol. 261, no. 2, pp. 377-380, 1999.

[18] D. Miklavcic, D. Semrov, H. Mekid, and L. M. Mir, "A validated model of in vivo electric field distribution in tissues for electrochemotherapy and for DNA electrotransfer for gene therapy," Biochimica Et Biophysica Acta, vol. 1523, no. 1, pp. 73-83, Sept. 2000.

[19] N. Pavšelj and D. Miklavčič, "Numerical modeling in electroporation-based biomedical applications," Radiol. Oncol., vol. 42, no. 3, pp. $159-168,2008$.

[20] R. V. Davalos, D. M. Otten, L. M. Mir, and B. Rubinsky, "Electrical impedance tomography for imaging tissue electroporation," IEEE Trans. Biomed. Eng., vol. 51, no. 5, pp. 761-767, May 2004.

[21] Y. Granot, A. Ivorra, E. Maor, and B. Rubinsky, "In vivo imaging of irreversible electroporation by means of electrical impedance tomography," Phys. Med. Biol., vol. 54, no. 16, pp. 4927-4943, 2009.

[22] D. Cukjati, D. Batiuskaite, F. André, D. Miklavcic, and L. M. Mir, "Real time electroporation control for accurate and safe in vivo nonviral gene therapy," Bioelectrochemistry, vol. 70, no. 2, pp. 501-507, 2007.

[23] M. Hjouj and B. Rubinsky, "Magnetic resonance imaging characteristics of nonthermal irreversible electroporation in vegetable tissue," $J$. Membrane Biol., vol. 236, no. 1, pp. 137-146, 2010.

[24] Y. Zhang et al., "MR imaging to assess immediate response to irreversible electroporation for targeted ablation of liver tissues: Preclinical feasibility studies in a rodent model," Radiology, vol. 256, no. 2, pp. 424-432, 2010.

[25] H. Gamba and D. Delpy, "Measurement of electrical current density distribution within the tissues of the head by magnetic resonance imaging," Med. Biol. Eng. Comput., vol. 36, no. 2, pp. 165-170, 1998.

[26] M. Joy, G. Scott, and M. Henkelman, "Invivo detection of applied electric currents by magnetic-resonance imaging," Magn. Reson. Imag., vol. 7, no. 1, pp. 89-94, 1989.

[27] G. Scott, M. Joy, R. Armstrong, and R. Henkelman, "Measurement of nonuniform current-density by magnetic-resonance," IEEE Trans. Med. Imag., vol. 10, no. 3, pp. 362-374, Sep. 1991.

[28] O. Kwon, E. Woo, J. Yoon, and J. Seo, "Magnetic resonance electrical impedance tomography (MREIT): Simulation study of J-substitution algorithm," IEEE Trans. Biomed. Eng., vol. 49, no. 2, pp. 160-167, Feb. 2002.

[29] E. J. Woo, S. Y. Lee, and C. W. Mun, "Impedance tomography using internal current density distribution measured by nuclear magnetic resonance," in Proc. SPIE, 1994, pp. 377-385.

[30] M. Eyuboglu, O. Birgul, and Y. Z. Ider, "A dual modality system for high resolution-True conductivity imaging," in Proc. XI Int. Conf. Elec. Bioimpedance (ICEBI), 2001, pp. 409-13.

[31] O. Kwon, J. Lee, and J. Yoon, "Equipotential line method for magnetic resonance electrical impedance tomography," Inverse Problems, vol. 18, no. 4, pp. 1089-1100, 2002.

[32] J. K. Seo, J.-R. Yoon, E. J. Woo, and O. Kwon, "Reconstruction of conductivity and current density images using only one component of magnetic field measurements," IEEE Trans. Biomed. Eng., vol. 50, no. 9, pp. 1121-1124, Sep. 2003.

[33] S. H. Oh et al., "Conductivity and current density image reconstruction using harmonic $\mathrm{Bz}$ algorithm in magnetic resonance electrical impedance tomography," Phys. Med. Biol., vol. 48, no. 19, pp. 3101-3116, 2003.

[34] H. J. Kim et al., "In vivo high-resolution conductivity imaging of the human leg using MREIT: The first human experiment," IEEE Trans. Med. Imag., vol. 28, no. 11, pp. 1681-1687, Nov. 2009.

[35] H. J. Kim et al., "In vivo electrical conductivity imaging of a canine brain using a 3 T MREIT system," Physiol. Measurement, vol. 29, no. 10, pp. 1145-1155, 2008.

[36] E. J. Woo, H. J. Kim, A. S. Minhas, Y. T. Kim, W. C. Jeong, and O. Kwon, "Electrical conductivity imaging of lower extremities using MREIT: Postmortem swine and in vivo human experiments," in Proc. Annu. Int. Conf. IEEE Eng. Med. Biol. Soc. Conf., 2008, pp. 5830-5833. 
[37] T. Kotnik, G. Pucihar, and D. Miklavčič, "Induced transmembrane voltage and its correlation with electroporation-mediated molecular transport," J. Membrane Biol., vol. 236, no. 1, pp. 3-13, 2010.

[38] I. Sersa, K. Beravs, N. J. Dodd, S. Zhao, D. Miklavcic, and F. Demsar, "Electric current density imaging of mice tumors," Magn. Reson. Med., vol. 37, no. 3, pp. 404-409, 1997.

[39] D. Haemmerich, D. J. Schutt, A. W. Wright, J. G. Webster, and D. M. Mahvi, "Electrical conductivity measurement of excised human metastatic liver tumours before and after thermal ablation," Physiol. Measurement, vol. 30, no. 5, pp. 459-466, 2009.

[40] S. Gabriel, R. W. Lau, and C. Gabriel, "The dielectric properties of biological tissues: II. Measurements in the frequency range $10 \mathrm{~Hz}$ to 20 GHz," Phys. Med. Biol., vol. 41, no. 11, pp. 2251-2269, 1996.

[41] S. Laufer, A. Ivorra, V. E. Reuter, B. Rubinsky, and S. B. Solomon, "Electrical impedance characterization of normal and cancerous human hepatic tissue," Physiol. Measurement, vol. 31, no. 7, pp. 995-1009, 2010.

[42] L. M. Mir, J. Gehl, and G. Sersa, "Standard operating procedures of the electrochemotherapy: Instructions for the use of bleomycin or cisplatin administered either systemically or local," Eur. J. Cancer (Suppl.), no. 4, pp. 14-25, 2006.

[43] I. Sersa, "Auxiliary phase encoding in multi spin-echo sequences: Application to rapid current density imaging," J. Magn. Reson., vol. 190, no. 1, pp. 86-94, 2008.

[44] J. Hennig, A. Nauerth, and H. Friedburg, "RARE imaging: A fast imaging method for clinical MR," Magn. Reson. Med., vol. 3, no. 6, pp. 823-833, 1986 .

[45] H. Khang et al., "J-substitution algorithm in magnetic resonance electrical impedance tomography (MREIT): Phantom experiments for static resistivity images," IEEE Trans. Med. Imag., vol. 21, no. 6, pp. 695-702, Jun. 2002.
[46] H. S. Nam, B. I. Lee, J. Choi, C. Park, and O. I. Kwon, "Conductivity imaging with low level current injection using transversal J-substitution algorithm in MREIT," Phys. Med. Biol., vol. 52, no. 22, pp. 6717-6730, 2007.

[47] S. Kim, O. Kwon, J. Seo, and J. Yoon, "On a nonlinear partial differential equation arising in magnetic resonance electrical impedance tomography," SIAM J. Math. Anal., vol. 34, no. 3, pp. 511-526, 2003.

[48] H. S. Nam and O. I. Kwon, "Axial anisotropic conductivity imaging based on projected current density in MREIT," IEEE Trans. Med. Imag., vol. 29, no. 3, pp. 781-789, Mar. 2010.

[49] H. Wang, Y. Wang, W. Yang, Z. Wang, and L. Hu, "Conductivity image reconstruction of oblique slice with c-shaped open permanent magnet MRI systems," IEEE Trans. Appl. Supercond., vol. 20, no. 3, pp. $814-817,2010$.

[50] C. Park, B. I. Lee, and O. I. Kwon, "Analysis of recoverable current from one component of magnetic flux density in MREIT and MRCDI," Phys. Med. Biol., vol. 52, no. 11, pp. 3001-3013, 2007.

[51] J. Seo, O. Kwon, B. Lee, and E. Woo, "Reconstruction of current density distributions in axially symmetric cylindrical sections using one component of magnetic flux density: Computer simulation study," Physiol. Measurement, vol. 24, no. 2, pp. 565-577, 2003.

[52] D. Miklavcic et al., "Towards treatment planning and treatment of deep-seated solid tumors by electrochemotherapy," Biomed. Eng. OnLine, vol. 9, no. 1, p. 10, 2010.

[53] L. Muftuler, M. Hamamura, O. Birgul, and O. Nalcioglu, "In vivo MRI electrical impedance tomography (MREIT) of tumors," Technol. Cancer Res. Treatment, vol. 5, no. 4, pp. 381-387, 2006.

[54] B. Kos, A. Zupanic, T. Kotnik, M. Snoj, G. Sersa, and D. Miklavcic, "Robustness of treatment planning for electrochemotherapy of deepseated tumors," J. Membrane Biol., vol. 236, no. 1, pp. 147-153, 2010. 